\title{
Finasteride and Its Potential for the Treatment of Female Pattern Hair Loss: Evidence to Date
}

This article was published in the following Dove Press journal:

Drug Design, Development and Therapy

\author{
Wimolsiri lamsumang (1D) \\ Kanchana Leerunyakul (D) \\ Poonkiat Suchonwanit (DD \\ Division of Dermatology, Department of \\ Medicine, Faculty of Medicine, Ramathibodi \\ Hospital, Mahidol University, Bangkok, \\ Thailand
}

\begin{abstract}
The currently approved treatment for female pattern hair loss (FPHL) includes topical minoxidil administration; however, this treatment fails to achieve hair regrowth in some patients. Finasteride, a selective $5 \alpha$-reductase inhibitor (5-ARI), may be considered as an alternative treatment. However, because of its potential teratogenic effects, clinical studies and use of finasteride for FPHL are limited. In this review, we aim to summarize the literature regarding the pharmacology, clinical efficacy, and adverse effects of oral finasteride for the treatment of FPHL and to provide novel therapeutic options including topical finasteride and dutasteride, a new generation 5-ARI, for the treatment of FPHL.
\end{abstract}

Keywords: alopecia, androgenetic alopecia, dutasteride, hair loss, topical finasteride, therapy

\section{Introduction}

Female pattern hair loss (FPHL) is a common hair condition in women characterized by diffuse hair thinning over the crown and parietal scalp with retention of the frontal hairline (Figure 1). ${ }^{1,2}$ The prevalence of FPHL increases with advancing age, affecting $50 \%$ of women during their lifetime. ${ }^{3}$ FPHL presents with follicular miniaturization and shortening of the anagen phase, similar to androgenetic alopecia (AGA) in men; nevertheless, the pathogenesis of FPHL remains unclear. ${ }^{4}$ The present understanding of relationship between androgenic hormone and FPHL is controversial as evidence suggests normal hormone levels in most balding females, and there is uncertainty regarding its hereditary nature. ${ }^{5}$

Various treatment options have been attempted to treat FPHL. The only agent approved by the US Food and Drug Administration (FDA) is topical minoxidil. ${ }^{6,7}$ Other treatment options currently available include low-level laser therapy, fractional laser therapy, platelet-rich plasma, human follicle stem cells and hair transplantation. $^{8-12}$ Nevertheless, the treatment outcome may not be satisfactory in some patients. Finasteride, an inhibitor of type II $5 \alpha$-reductase enzyme, is currently indicated for AGA in men. It has been increasingly used as an offlabel treatment for FPHL. ${ }^{13}$ Despite its potential teratogenic effect, several publications on finasteride in FPHL have shown positive results. Therefore, this review aims to summarize the pharmacology, therapeutic efficacy as well as safety of oral finasteride for the treatment of FPHL. Furthermore, we provide novel therapeutic options of $5 \alpha$-reductase inhibitor (5-ARI), namely topical finasteride and oral dutasteride.
Correspondence: Poonkiat Suchonwanit Email poonkiat@hotmail.com 


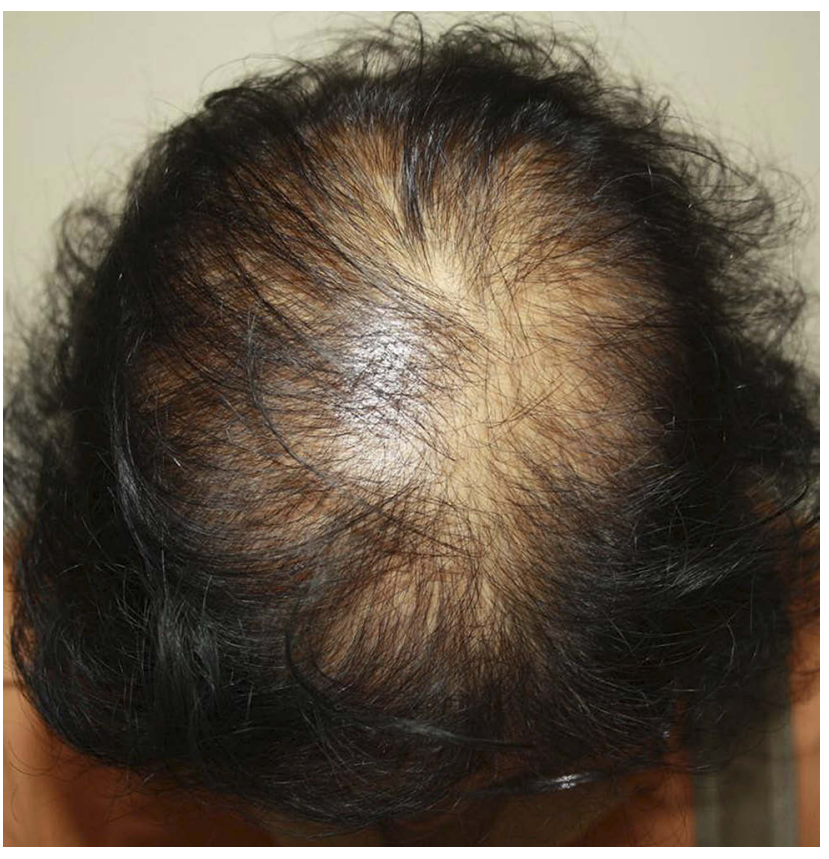

Figure I Female pattern hair loss: hair thinning mostly confined to the crown with retention of frontal hairline.

\section{Pharmacology of Finasteride}

Finasteride is a synthetic 4-azasteroid compound $\left(\mathrm{C}_{23} \mathrm{H}_{36} \mathrm{~N}_{2} \mathrm{O}_{2}\right)$ (Figure 2) that competitively inhibits type II $5 \alpha$-reductase, resulting in the prevention of the conversion of testosterone to dihydrotestosterone (DHT) in the skin, liver and prostate gland. ${ }^{14} \mathrm{~A}$ study showed that oral finasteride reaches its maximum plasma concentration approximately 1-2 hrs after ingestion, while achieving the steady-state within three days. Finasteride suppresses scalp DHT levels by $43 \%$ at 28 days, up to $65 \%$ at 42 days of treatment with finasteride $5 \mathrm{mg}$ daily in patients with AGA. $^{15}$ To our knowledge, no study evaluating the changes of scalp DHT in women with FPHL has been reported. The bioavailability of finasteride is $80 \%$ and it

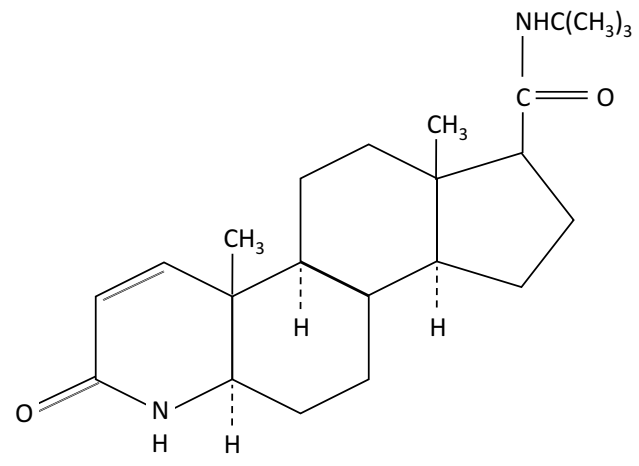

Figure 2 Chemical structure of finasteride. is not meaningfully influenced by food. ${ }^{16}$ Finasteride can cross the blood-brain barrier; however, it only insignificantly distributes to cerebrospinal fluid. ${ }^{17}$ Finasteride has no effect on luteinizing hormone ( $\mathrm{LH})$ and folliclestimulating hormone (FSH), while it slightly increases circulating testosterone levels but remains within the normal physiologic range. ${ }^{18}$

Finasteride is exclusively metabolized via cytochrome P450 3A4 in the liver. ${ }^{19}$ The major metabolites ( $\omega$ hydroxyfinasteride and a monocarboxylic acid derivative) contain less than $20 \%$ of $5 \alpha$-reductase inhibitory effect and are eliminated through bile. ${ }^{17}$ After 7 days of administration, approximately $57 \%$ of the dose is excreted in the feces, and approximately $39 \%$ is excreted in the urine. ${ }^{17}$ DHT returns to pretreatment levels by approximately 14 days after cessation. ${ }^{20}$ Despite a lack of studies in patients with abnormal liver functions, it is recommended that finasteride be used with caution in patients with hepatic impairment as it is preferentially metabolized in the liver. In healthy young adults, the mean half-life of finasteride in plasma is $6 \mathrm{hrs}$. The mean elimination half-life in elderly subjects (age $\geq 70$ years) is approximately $8 \mathrm{hrs,} \mathrm{not} \mathrm{sig-}$ nificantly different from that of subjects who are 45-60 years of age. ${ }^{21}$ Therefore, no dosage adjustment is required for the elderly. Although finasteride is primarily metabolized by hepatic enzymes, it does not appear to interfere with the enzyme system. Interaction studies showed that there are no drug interactions between finasteride and antipyrine, digoxin, propranolol, theophylline, and warfarin. Moreover, there is no evidence of clinically significant interactions with concomitant use of drugs such as anti-convulsants and nonsteroidal anti-inflammatory drugs. ${ }^{17}$

Finasteride is classified as pregnancy category $\mathrm{X}$, ie, it is contradicted in women who are or may become pregnant. ${ }^{17}$ An animal study showed that finasteride led to dose-dependent development of hypospadias in male offspring, and that the abnormal development of external genitalia is an expected aftereffect from inhibition of type II $5 \alpha$-reductase similar to male children with genetic $5 \alpha-$ reductase deficiency. ${ }^{22}$ No developmental abnormalities were seen in female fetuses. Finasteride is also prohibited in lactating women because of its potential risks in male infants, despite the unavailability of data on its excretion in human milk. Finasteride can interfere with the estrogen/ testosterone balance, leading to potential risk of estrogenmediated malignant transformation; it should be avoided in those who have family history of breast cancer. ${ }^{23}$ 


\section{Therapeutic Efficacy of Finasteride in Female Pattern Hair Loss}

Finasteride has been commonly used in men with AGA and benign prostatic hyperplasia, and is progressively prescribed in women with hyperandrogenism-associated conditions including acne vulgaris, hirsutism and pattern alopecia induced by testosterone in female-to-male transgender patients. ${ }^{13,24}$ Nevertheless, studies of the efficacy of oral finasteride in FPHL are limited because of the potential risk of teratogenicity in the male fetus. ${ }^{5}$ The dosage of finasteride for FPHL ranges from 1 to $5 \mathrm{mg}$ daily. Case reports and series using various regimens showed increased hair density and reduction of hair shedding in pre- and postmenopausal women with or without hyperandrogenism. ${ }^{25-29}$ Evidence from clinical trials of finasteride in FPHL is summarized in Table 1.

A multicenter, double-blind, randomized controlled trial (RCT) of 137 postmenopausal women with mild-tomoderate FPHL reported no significant differences in changes of hair loss by hair count between finasteride $1 \mathrm{mg}$ and placebo after 1 year of treatment. ${ }^{3}$ Patient and investigator assessments, as well as global photographic assessment (GPA), failed to show any significant improvement from baseline with respect to hair growth, hair appearance or stabilization of hair loss in both groups. Histological examination of scalp biopsy specimens correspondingly showed no significant differences between the groups with respect to alterations from baseline regarding terminal-to-vellus hair ratio and anagen-to-telogen ratio. ${ }^{30,31}$

A subsequent open-label, uncontrolled study using lowdose finasteride $(1.25 \mathrm{mg})$ in 18 normoandrogenic FPHL patients similarly failed to demonstrate increasing hair density evaluated by phototrichogram. ${ }^{32}$ No clinical response was observed by patient self-assessment and physician's GPA compared to baseline. In contrast to these failures, a retrospective cohort demonstrated the efficacy of finasteride $1.25 \mathrm{mg}$ or dutasteride $0.15 \mathrm{mg}$ daily in $120 \mathrm{FPHL}$ patients. ${ }^{33}$ Each group contained 60 patients, half of whom were aged 50 years or above. After 3 years of treatment, $82 \%$ of patients in the finasteride group and $83 \%$ of patients in dutasteride group demonstrated improvement in hair thickness compared to the baseline. GPA by physicians at the vertex and center of the scalp reported increased hair density and scalp coverage by $67 \%$ in the finasteride group and $66 \%$ in the dutasteride group. Nevertheless, the study did not objectively measure hair density or compare the effectiveness with placebo.
Medium-dose finasteride ( $2.5 \mathrm{mg}$ daily) also proved to be efficacious in normoandrogenic postmenopausal women with FPHL. Trueb et al conducted a small prospective study using finasteride $2.5 \mathrm{mg}$ daily in four women and $5 \mathrm{mg}$ daily in one woman who had been previously treated with topical minoxidil alone or combined with cyproterone acetate (CPA), topical estradiol, and systemic estrogens without clinical response. ${ }^{34}$ By 6 months, all patients reported the stabilization of hair loss and four patients self-reported noticeable hair growth. These findings were confirmed by investigator assessments and photography evaluations. A single-center, uncontrolled, prospective trial further evaluated the efficacy of the medium-dose finasteride in 37 pre-menopausal women with FPHL in the absence of hyperandrogenism. ${ }^{30}$ Finasteride was given for 12 months at a dose of $2.5 \mathrm{mg}$ daily with concomitant use of an oral contraceptive containing drospirenone and ethinyl estradiol to prevent pregnancy. After 12 months, global photography displayed the enhancement of hair density in $62 \%$ of patients. Hair density scores assessed by computerized videodermoscopy significantly increased compared to baseline in $32 \%$ of patients. On self-administered questionnaire, $78 \%$ of the patients reported improvement, while $22 \%$ reported stabilization of hair loss. Nonetheless, the clinical outcome of $2.5 \mathrm{mg}$ finasteride was inconclusive as improvement in hair density may have resulted from the antiandrogenic effect of the oral contraceptive.

High-dose finasteride ( $5 \mathrm{mg}$ daily) has been investigated for efficacy in treatment of FPHL. A retrospective study using self-filled questionnaires evaluated effectiveness of finasteride $5 \mathrm{mg}$ daily for unknown duration in 12 patients with FPHL or acne vulgaris. ${ }^{35}$ Five of six patients with FPHL reported to benefit from the treatment. Carmina et al conducted a subsequent single-center, openlabel, RCT in hyperandrogenic pre-menopausal women with FPHL. ${ }^{36}$ Forty-eight patients were randomly assigned into four groups which consisted of finasteride $5 \mathrm{mg}$ daily, CPA $50 \mathrm{mg}$ (day 5-15 of menstrual cycle) with ethinyl estradiol $25 \mu \mathrm{g} /$ day (day 5-25 of menstrual cycle), flutamide $250 \mathrm{mg}$ daily, and no treatment for 1 year. All patients including controls showed decreases in Ludwig scores at the end of treatment. Flutamide was the only agent that gave a statistically significant reduction of Ludwig scores and provided the greatest clinical response in terms of slowing hair loss as assessed by the patients. Using a 7-point scale, flutamide demonstrated the best, although not significant, improved hair growth compared 


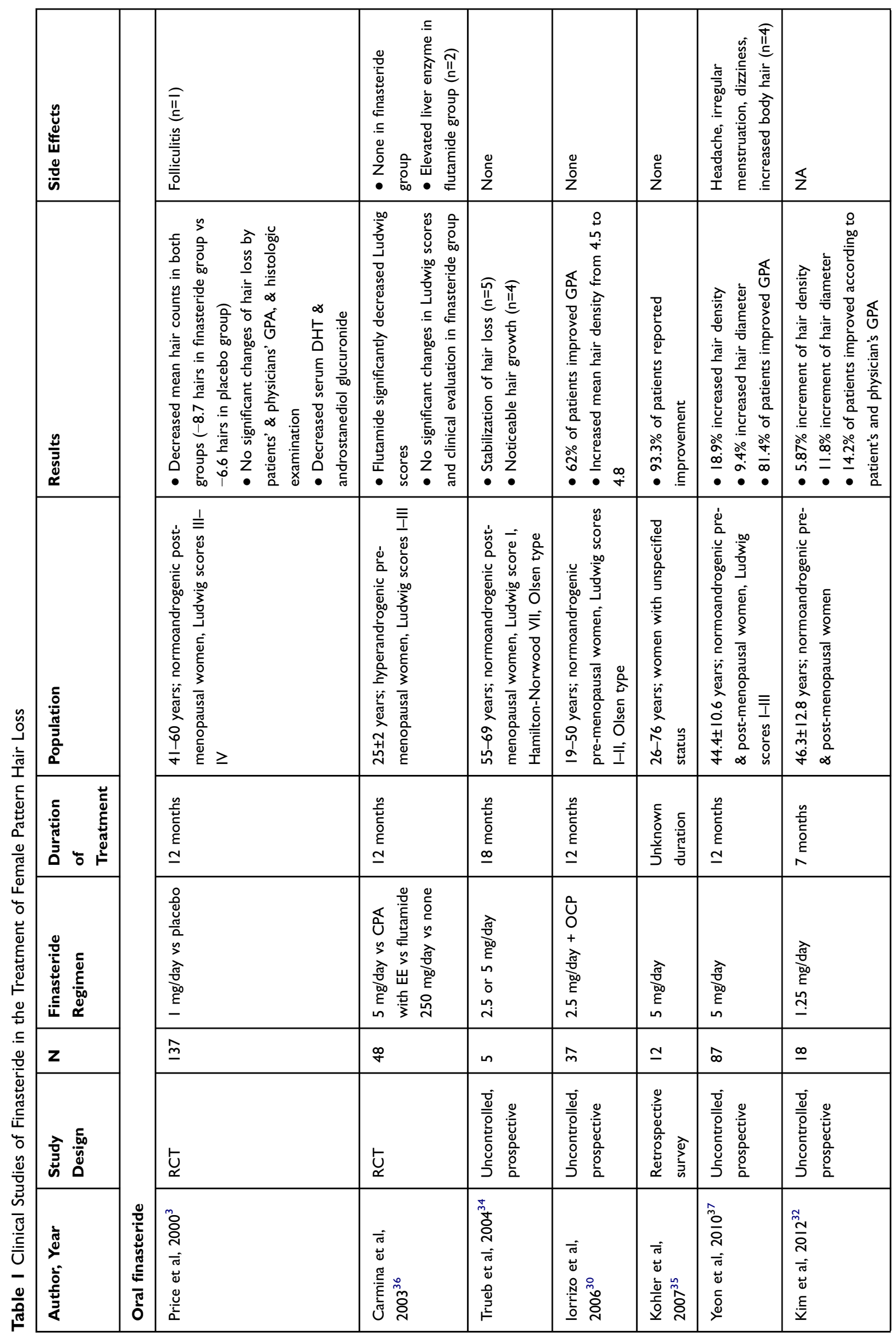




\begin{tabular}{|c|c|c|c|c|}
\hline 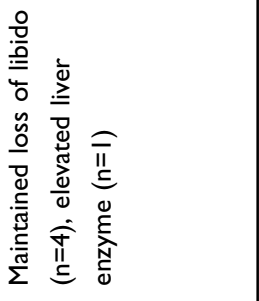 & $\S$ & & $\begin{array}{l}\text { I } \\
\text { Oे }\end{array}$ & 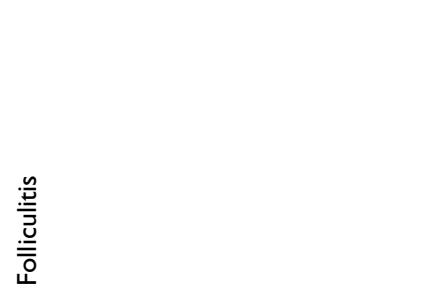 \\
\hline 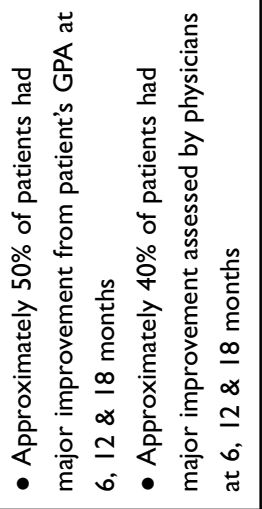 & 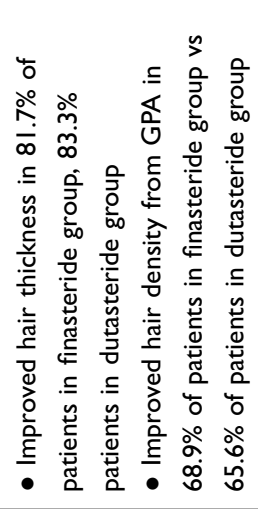 & & 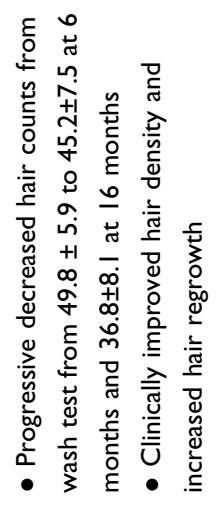 & 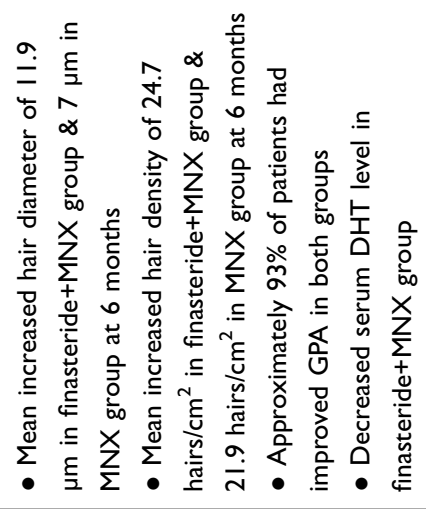 \\
\hline 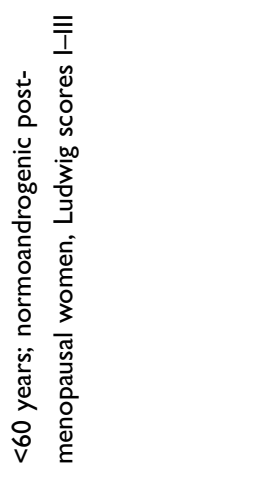 & 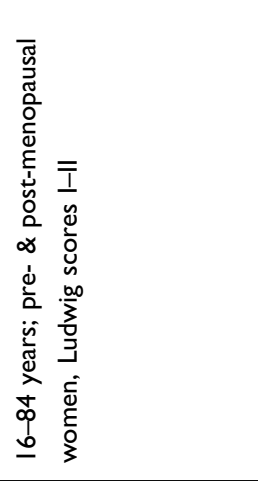 & & 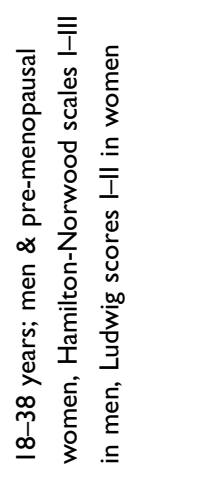 & 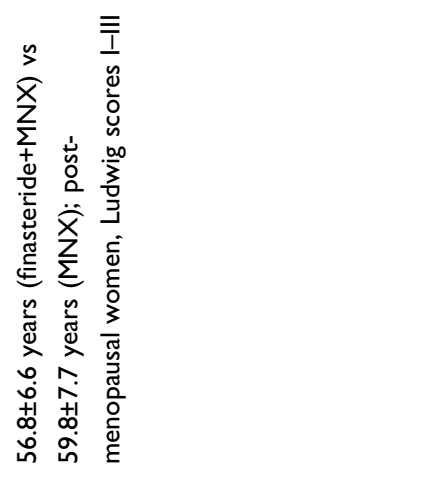 \\
\hline 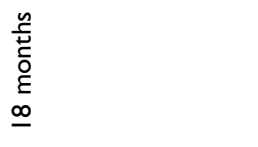 & $\begin{array}{l}\text { 号 } \\
\text { న్ } \\
m\end{array}$ & & 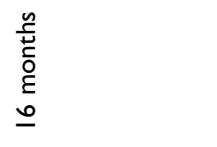 & 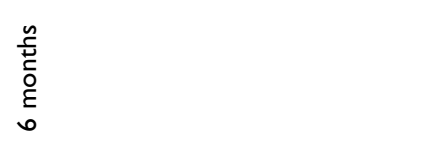 \\
\hline $\begin{array}{l}\frac{\widehat{\vec{J}}}{0} \\
\text { है } \\
\text { हn }\end{array}$ & 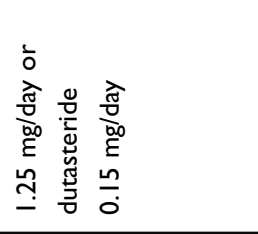 & & 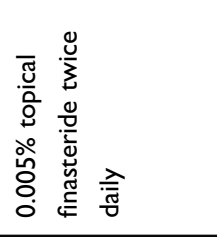 & 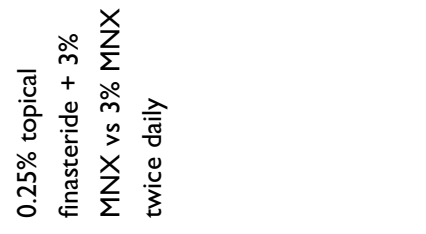 \\
\hline 우 & $\stackrel{\text { I }}{ }$ & & กิ & 이 \\
\hline 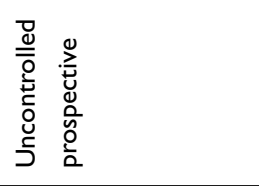 & 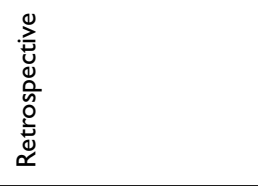 & & 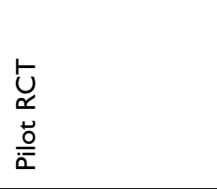 & $\underset{\swarrow}{\breve{x}}$ \\
\hline 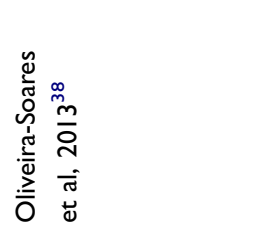 & 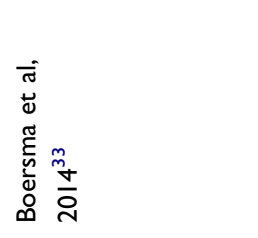 & 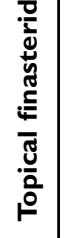 & 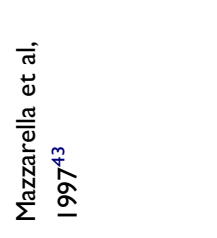 & 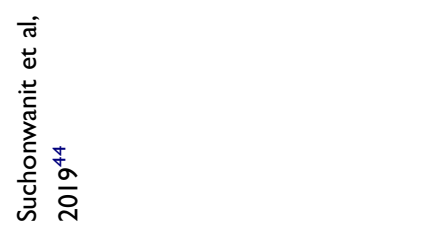 \\
\hline
\end{tabular}


to CPA, finasteride, and controls. Two patients had elevated liver transaminase from flutamide, while no adverse effects were noted in the finasteride and CPA groups. The authors concluded that finasteride was ineffective for treatment of FPHL.

By contrast, a non-randomized, uncontrolled study of high-dose finasteride in 87 normoandrogenic, pre- and postmenopausal women with FPHL reported clinical improvement. $^{37}$ Eighty-six patients completed 12 months of treatment with finasteride $5 \mathrm{mg}$ daily, whereas one patient discontinued because of headache. At the end of the study, phototrichograms demonstrated significantly increased hair density by $18.9 \%$ and hair diameter by $9.4 \%$ compared to baseline. GPA showed clinical improvement in $81 \%$ of patients. There was no significant difference in the clinical response between pre- and post-menopausal groups. The authors concluded that finasteride might be effective and safe for FPHL in women without hyperandrogenism. Another uncontrolled prospective study on efficacy of finasteride $5 \mathrm{mg}$ daily was conducted in 40 normoandrogenic post-menopausal FPHL patients. All patients were divided into three age groups: $<60(\mathrm{n}=22), 60-70(\mathrm{n}=13)$, and $>70$ years $(n=5) .{ }^{38}$ Fifty-five percent of patients reported having major improvement, and 30\% reported having moderate improvement with as little as 6 months of treatment. Although the improvement tendency was observed over time, it was relatively constant over 12 and 18 monthperiods. Patients who were $>70$ years of age demonstrated poorer clinical responses than did the other two younger groups. According to pre-existing data, high-dose finasteride appears to be more efficacious than the lower doses for the treatment of FPHL.

\section{Mechanism of Finasteride in Female Pattern Hair Loss}

Because FPHL is a complex disease, the mechanism by which finasteride improves hair loss is not well established. A study in mice proposed that DHT may downgrade insulin-like growth factor-1 (IGF-1) expression by inhibiting the release of calcitonin gene-related peptide that interacts with the androgen receptors, thereby preventing hair growth. ${ }^{39}$ These findings suggested that finasteride might be associated with increasing IGF-1 production in the dermal papillae by decreasing DHT levels. ${ }^{40}$ Rushton et al found that finasteride administration resulted in increased mean hair density without insignificant changes of vellus hair counts. ${ }^{41}$ The observed hair regrowth in
FPHL may be supported by reactivating telogen/kenogen follicles into anagen follicles rather than vellus-to-terminal hair transformation. Further studies are needed to determine the mechanism of finasteride in FPHL. Concerning contradictory efficacy in pre-existing literatures, oral finasteride may be served as an alternative modality in FPHL patients who fail minoxidil treatment. We suggest using finasteride in postmenopausal women to avoid potential teratogenic effects.

\section{New Formulation of Finasteride}

A topical formulation has been proposed as a new treatment modality to minimize the unwanted systemic side effects of oral finasteride, particularly in child-bearing aged women. The ideal formulation of topical finasteride is high skin penetration and low systemic absorption. Topical finasteride has recently shown to be effective in males with AGA. ${ }^{42}$ Nevertheless, studies on efficacy of topical finasteride in females have been limited. Mozzarella et al conducted a placebo-controlled trial with $0.005 \%$ finasteride solution twice daily for 16 months in 52 patients, including 28 men and 24 pre-menopausal women. ${ }^{43}$ The finasteride-treated group showed a significantly greater reduction of hair shedding from the 6th month of treatment and progressively continued throughout the 16-month period. At the end of study, some finasteride-treated scalps demonstrated gradual increases in hair density.

A recent single-center, double-blind RCT compared the efficacy between topical 3\% minoxidil solution alone and in combination with $0.25 \%$ finasteride for treatment of 30 postmenopausal FPHL patients. ${ }^{44}$ The combined treatment group showed significantly superior results compared to the minoxidil monotherapy group in terms of increased hair diameter at weeks 24 , while increased hair density was progressively observed over time from weeks 8 in both groups with similar time-courses. At week 24, clinical improvements as assessed by GPA from blinded dermatologists and participants were reported in approximately $93 \%$ of participants in the combined treatment group but were not significantly different from the minoxidil group. The authors agreed with the previous literature that adding $0.25 \%$ finasteride to minoxidil solution showed evidence of systemic absorption by lowering serum DHT level after 24 weeks of treatment. Nevertheless, DHT levels remained in the normal range throughout the study.

Topical application with finasteride is a promising treatment for FPHL with favorable results. Many aspects of topical finasteride are required for further investigations, 
including delivery systems, concentrations, and regimens. Because several studies used various formulations, a comparative study between each formulation (eg, gel versus solution) should be performed to determine the best use of topical finasteride.

\section{Tolerability of Finasteride}

Side effects of finasteride have been relatively well documented in male AGA patients. These side effects include ejaculation disorders, decreased libido, erectile dysfunction, breast tenderness and enlargement, dizziness, allergic reactions, increased liver enzymes, and depression. ${ }^{45,46}$ Post-finasteride syndrome, characterized by persistent sexual, neurological, and physical adverse reactions, has also been described. ${ }^{45,47}$ Because finasteride is not widely used in the treatment of FPHL, the side effects in women are not well elucidated.

Oral finasteride given at low-to-medium dose did not demonstrate any complications. Oral finasteride $1 \mathrm{mg}$ daily reduced serum levels of DHT and androstanediol glucuronide in postmenopausal women without clinical change, while had no effect on other hormones including serum testosterone, LH, FSH, thyroid-stimulating hormone and prolactin. High-dose oral finasteride disturbed women with side effects analogous to those of men. In five patients, Kohler et al found one patient with decreased libido, one with dry skin and one with mild acne. ${ }^{35}$ In a cohort study of 87 patients, headache, irregular menstruation, dizziness and increased body hair growth were reported in four patients, but were minimal and transient. ${ }^{37}$ Another cohort study by Oliveira-Soares et al noted persistent libido reduction in four patients and increased liver enzyme levels in one patient. ${ }^{38}$

By contrast, no systemic side effects have been reported with the use of topical finasteride in FPHL patients except suppression of serum DHT levels. Minimal local side effects such as pruritus and irritation have been reported, but with an excellent tolerability. ${ }^{43,44}$ Because of limited data, further clinical trials with longterm use should be performed to confirm the safety of topical finasteride in women.

\section{New Generation of $5 \alpha-$-Reductase Inhibitors}

Dutasteride is a second-generation 5-ARI that inhibits both type I and II $5 \alpha$-reductase isoenzymes. It is three times more potent at inhibiting type I enzyme and 100 times more potent at inhibiting type II enzyme than is finasteride. ${ }^{48}$ Although dutasteride is not currently FDA-approved for pattern hair loss, recent studies have pointed to its ability to serve as a therapeutic agent. ${ }^{49,50}$ A study in 416 men with AGA found that dutasteride $2.5 \mathrm{mg}$ daily had efficacy superior to that of finasteride $5 \mathrm{mg}$ daily and placebo in terms of increasing hair counts and scalp appearance by GPA after 12 and 24 weeks of treatment. ${ }^{49}$ By contrast, pre-existing studies of dutasteride for treatment of FPHL are limited. A case study of a 46-year-old woman with FPHL who had little response to minoxidil and finasteride reported clinical improvement after 6 months of dutasteride $0.5 \mathrm{mg}$ daily and marked improvement after 9 months of therapy. ${ }^{51}$ Boersma et al found that dutasteride $0.15 \mathrm{mg}$ daily was more effective than finasteride $1.25 \mathrm{mg}$ daily as it caused higher mean hair thickness at the vertex and the center of the scalp in patients younger than 50 years. ${ }^{33}$ These available data support the efficacy of dutasteride for the treatment of FPHL; nevertheless, further studies comparing dutasteride with finasteride and placebo are necessary to determine its real safety and efficacy.

\section{Conclusion}

This review summarizes the pharmacology and presents potential uses of finasteride in women, especially in the postmenopausal-aged group, and those wishing to avoid systemic side effects. Dutasteride, a new generation of 5-ARIs is also discussed. The studies we reviewed suggest that finasteride could be considered as an alternative treatment option for FPHL. Nevertheless, many questions regarding finasteride, particularly with respect to topical formulations, are yet to be answered. Further studies are encouraged to determine the maximum therapeutic efficacy and to evaluate the consequences of finasteride use in FPHL.

\section{Statement of Ethics}

The patient provided written informed consent to perform all necessary investigations, to take clinical photographs, and to use them for research purposes and publication.

\section{Disclosure}

The authors report no conflicts of interest in this work.

\section{References}

1. Olsen EA. Female pattern hair loss. J Am Acad Dermatol. 2001;45(3 Suppl):S70-80. doi:10.1067/mjd.2001.117426

2. Rojhirunsakool S, Suchonwanit P. Parietal scalp is another affected area in female pattern hair loss: an analysis of hair density and hair diameter. Clin Cosmet Investig Dermatol. 2018;11:7-12. doi:10.21 47/CCID.S153768 
3. Price VH, Roberts JL, Hordinsky M, et al. Lack of efficacy of finasteride in postmenopausal women with androgenetic alopecia. J Am Acad Dermatol. 2000;43(5):768-776. doi:10.1067/mjd.2000.107953

4. Olsen EA, Messenger AG, Shapiro J, et al. Evaluation and treatment of male and female pattern hair loss. J Am Acad Dermatol. 2005;52 (2):301-311. doi:10.1016/j.jaad.2004.04.008

5. Stout SM, Stumpf JL. Finasteride treatment of hair loss in women. Ann Pharmacother. 2010;44(6):1090-1097. doi:10.1345/aph.1M591

6. Rogers NE, Avram MR. Medical treatments for male and female pattern hair loss. J Am Acad Dermatol. 2008;59(4):547-566; quiz 567-548. doi:10.1016/j.jaad.2008.07.001

7. Suchonwanit P, Thammarucha S, Leerunyakul K. Minoxidil and its use in hair disorders: a review. Drug Des Devel Ther. 2019;13:2777-2786. doi:10.2147/DDDT.S214907

8. Suchonwanit P, Chalermroj N, Khunkhet S. Low-level laser therapy for the treatment of androgenetic alopecia in Thai men and women: a 24-week, randomized, double-blind, sham device-controlled trial. Lasers Med Sci. 2019;34(6):1107-1114. doi:10.1007/s10103-01802699-9

9. Lee GY, Lee SJ, Kim WS. The effect of a $1550 \mathrm{~nm}$ fractional erbium-glass laser in female pattern hair loss. J Eur Acad Dermatol Venereol. 2011;25 (12):1450-1454. doi:10.1111/j.1468-3083.2011.04183.x

10. Suchonwanit P, Rojhirunsakool S, Khunkhet S. A randomized, investigator-blinded, controlled, split-scalp study of the efficacy and safety of a 1550-nm fractional erbium-glass laser, used in combination with topical 5\% minoxidil versus 5\% minoxidil alone, for the treatment of androgenetic alopecia. Lasers Med Sci. 2019;34 (9):1857-1864. doi:10.1007/s10103-019-02783-8

11. Gentile P, Cole JP, Cole MA, et al. Evaluation of not-activated and activated PRP in hair loss treatment: role of growth factor and cytokine concentrations obtained by different collection systems. Int J Mol Sci. 2017;18(2):E408. doi:10.3390/ijms18020408

12. Gentile P. Autologous cellular method using micrografts of human adipose tissue derived follicle stem cells in androgenic alopecia. Int J Mol Sci. 2019;20(14):E3446. doi:10.3390/ijms20143446

13. Hu AC, Chapman LW, Mesinkovska NA. The efficacy and use of finasteride in women: a systematic review. Int J Dermatol. 2019;58 (7):759-776. doi:10.1111/ijd.2019.58.issue-7

14. van Zuuren EJ, Fedorowicz Z, Schoones J. Interventions for female pattern hair loss. Cochrane Database Syst Rev. 2016;5(5):Cd007628.

15. Dallob AL, Sadick NS, Unger W, et al. The effect of finasteride, a 5 alpha-reductase inhibitor, on scalp skin testosterone and dihydrotestosterone concentrations in patients with male pattern baldness. J Clin Endocrinol Metab. 1994;79(3):703-706. doi:10.1210/jcem.79.3.8077 349

16. Ohtawa M, Morikawa H, Shimazaki J. Pharmacokinetics and biochemical efficacy after single and multiple oral administration of N-(2-methyl-2-propyl)-3-oxo-4-aza-5 alpha-androst-1-ene-17 beta-carboxamide, a new type of specific competitive inhibitor of testosterone 5 alpha-reductase, in volunteers. Eur J Drug Metab Pharmacokinet. 1991;16(1):15-21. doi:10.1007/BF03189869

17. Carlin JR, Hoglund P, Eriksson LO, et al. Disposition and pharmacokinetics of $[14 \mathrm{C}]$ finasteride after oral administration in humans. Drug Metab Dispos. 1992;20(2):148-155.

18. Matzkin H, Chen J, Lewyshon O, Ayalon D, Braf Z. Effects of long term treatment with finasteride (MK-906), a 5-alpha reductase inhibitor, on circulating LH, FSH, prolactin and estradiol. Horm Metab Res. 1992;24(10):498-499. doi:10.1055/s-2007-1003373

19. Hulin-Curtis SL, Petit D, Figg WD, Hsing AW, Reichardt JK. Finasteride metabolism and pharmacogenetics: new approaches to personalized prevention of prostate cancer. Future Oncol. 2010;6 (12):1897-1913. doi:10.2217/fon.10.149

20. Gormley GJ, Stoner E, Rittmaster RS, et al. Effects of finasteride (MK-906), a 5 alpha-reductase inhibitor, on circulating androgens in male volunteers. J Clin Endocrinol Metab. 1990;70(4):1136-1141. doi:10.1210/jcem-70-4-1136
21. Gregoire S, Winchell GA, Constanzer M, et al. Multiple dose pharmacokinetics of finasteride, a 5a-reductase inhibitor in men 45-60 and ${ }^{3} 70$ years old [abstract]. Pharm Res. 1990;7:S-53.

22. Prahalada S, Tarantal AF, Harris GS, et al. Effects of finasteride, a type 2 5-alpha reductase inhibitor, on fetal development in the rhesus monkey (Macaca mulatta). Teratology. 1997;55(2):119-131. doi:10.1002/(ISSN)1096-9926

23. Shenoy NK, Prabhakar SM. Finasteride and male breast cancer: does the MHRA report show a link? J Cutan Aesthet Surg. 2010;3 (2):102-105. doi:10.4103/0974-2077.69022

24. Moreno-Arrones OM, Becerra A, Vano-Galvan S. Therapeutic experience with oral finasteride for androgenetic alopecia in female-to-male transgender patients. Clin Exp Dermatol. 2017;42 (7):743-748. doi:10.1111/ced.2017.42.issue-7

25. Thai KE, Sinclair RD. Finasteride for female androgenetic alopecia. Br J Dermatol. 2002;147(4):812-813. doi:10.1046/j.1365-2133.2002. 49084.x

26. Valsecchi R, Leghissa P, Riva M. Female androgenetic alopecia treated by finasteride: a case forward. Acta Derm Venereol. 2004;84 (6):488-489.

27. Hong JB, Chiu HC, Chan JY, Chen RJ, Lin SJ. A woman with iatrogenic androgenetic alopecia responding to finasteride. $\mathrm{Br} J$ Dermatol. 2007;156(4):754-755. doi:10.1111/j.1365-2133.2006.07719.x

28. Boychenko O, Bernstein RM, Schweiger ES. Finasteride in the treatment of female pattern (androgenic) alopecia: a case report and review of the literature. Cutis. 2012;90(2):73-76.

29. Shum KW, Cullen DR, Messenger AG. Hair loss in women with hyperandrogenism: four cases responding to finasteride. $\mathrm{J} \mathrm{Am} \mathrm{Acad}$ Dermatol. 2002;47(5):733-739. doi:10.1067/mjd.2002.124608

30. Iorizzo M, Vincenzi C, Voudouris S, Piraccini BM, Tosti A. Finasteride treatment of female pattern hair loss. Arch Dermatol. 2006;142(3):298-302. doi:10.1001/archderm.142.3.298

31. Whiting DA, Waldstreicher J, Sanchez M, Kaufman KD. Measuring reversal of hair miniaturization in androgenetic alopecia by follicular counts in horizontal sections of serial scalp biopsies: results of finasteride $1 \mathrm{mg}$ treatment of men and postmenopausal women. J Investig Dermatol Symp Proc. 1999;4(3):282-284. doi:10.1038/sj. jidsp. 5640230

32. Kim WJ, Song M, Ko HC, Kim BS, Kim MB. Efficacy of finasteride $1.25 \mathrm{mg}$ on female pattern hair loss; pilot study. Ann Dermatol. 2012;24(3):370-372. doi:10.5021/ad.2012.24.3.370

33. Boersma IH, Oranje AP, Grimalt R, Iorizzo M, Piraccini BM, Verdonschot EH. The effectiveness of finasteride and dutasteride used for 3 years in women with androgenetic alopecia. Indian J Dermatol Venereol Leprol. 2014;80(6):521-525. doi:10.4103/0378-6323.144162

34. Trueb RM. Finasteride treatment of patterned hair loss in normoandrogenic postmenopausal women. Dermatology. 2004;209 (3):202-207. doi:10.1159/000079890

35. Kohler C, Tschumi K, Bodmer C, Schneiter M, Birkhaeuser M. Effect of finasteride $5 \mathrm{mg}$ (Proscar) on acne and alopecia in female patients with normal serum levels of free testosterone. Gynecol Endocrinol. 2007;23(3):142-145. doi:10.1080/09513590701214463

36. Carmina E, Lobo RA. Treatment of hyperandrogenic alopecia in women. Fertil Steril. 2003;79(1):91-95. doi:10.1016/S00150282(02)04551-X

37. Yeon JH, Jung JY, Choi JW, et al. $5 \mathrm{mg}$ /day finasteride treatment for normoandrogenic Asian women with female pattern hair loss. J Eur Acad Dermatol Venereol. 2011;25(2):211-214. doi:10.1111/jdv.2010.25.issue-2

38. Oliveira-Soares R, e Silva JM, Correia MP, Andre MC. Finasteride $5 \mathrm{mg} /$ day treatment of patterned hair loss in normo-androgenetic postmenopausal women. Int $J$ Trichol. 2013;5(1):22-25. doi:10. 4103/0974-7753.114709

39. Zhao J, Harada N, Okajima K. Dihydrotestosterone inhibits hair growth in mice by inhibiting insulin-like growth factor-I production in dermal papillae. Growth Horm IGF Res. 2011;21(5):260-267. doi:10.1016/j.ghir.2011.07.003 
40. Tang L, Bernardo O, Bolduc C, Lui H, Madani S, Shapiro J. The expression of insulin-like growth factor 1 in follicular dermal papillae correlates with therapeutic efficacy of finasteride in androgenetic alopecia. J Am Acad Dermatol. 2003;49(2):229-233. doi:10.1067/ S0190-9622(03)00777-1

41. Hugh Rushton D, Norris MJ, Van Neste D. Hair regrowth in male and female pattern hair loss does not involve the conversion of vellus hair to terminal hair. Exp Dermatol. 2016;25:482-484. doi:10.1111/ exd. 12945

42. Suchonwanit P, Srisuwanwattana P, Chalermroj N, Khunkhet S. A randomized, double-blind controlled study of the efficacy and safety of topical solution of $0.25 \%$ finasteride admixed with $3 \%$ minoxidil vs. $3 \%$ minoxidil solution in the treatment of male androgenetic alopecia. J Eur Acad Dermatol Venereol. 2018;32 (12):2257-2263. doi:10.1111/jdv.2018.32.issue-12

43. Mazzarella GF, Loconsole GF, Cammisa GA, Mastrolonardo GM, Vena GA. Topical finasteride in the treatment of androgenetic alopecia: preliminary evaluations after a 16-month therapy course. J Dermatol Treat. 1997;8:189-192. doi:10.3109/09546639709160 517

44. Suchonwanit P, Iamsumang W, Rojhirunsakool S. Efficacy of topical combination of $0.25 \%$ finasteride and $3 \%$ minoxidil versus $3 \%$ minoxidil solution in female pattern hair loss: a randomized, double-blind, controlled study. Am J Clin Dermatol. 2019;20 (1):147-153. doi:10.1007/s40257-018-0387-0
45. Seale LR, Eglini AN, McMichael AJ. Side effects related to 5 alpha-reductase inhibitor treatment of hair loss in women: a review. J Drugs Dermatol. 2016;15(4):414-419.

46. McClellan KJ, Markham A. Finasteride: a review of its use in male pattern hair loss. Drugs. 1999;57(1):111-126. doi:10.2165/00003495199957010-00014

47. Gupta AK, Sharma N, Shukla P. Atypical post-finasteride syndrome: a pharmacological riddle. Indian J Pharmacol. 2016;48(3):316-317. doi:10.4103/0253-7613.182898

48. Clark RV, Hermann DJ, Cunningham GR, Wilson TH, Morrill BB, Hobbs S. Marked suppression of dihydrotestosterone in men with benign prostatic hyperplasia by dutasteride, a dual 5alpha-reductase inhibitor. $J$ Clin Endocrinol Metab. 2004;89(5):2179-2184. doi: $10.1210 /$ jc.2003-030330

49. Olsen EA, Hordinsky M, Whiting D, et al. The importance of dual 5alpha-reductase inhibition in the treatment of male pattern hair loss: results of a randomized placebo-controlled study of dutasteride versus finasteride. J Am Acad Dermatol. 2006;55(6):1014-1023. doi:10.1016/j.jaad.2006.05.007

50. Gupta AK, Charrette A. The efficacy and safety of 5alpha-reductase inhibitors in androgenetic alopecia: a network meta-analysis and benefit-risk assessment of finasteride and dutasteride. J Dermatolog Treat. 2014;25(2):156-161. doi:10.3109/09546634.2013.813011

51. Olszewska M, Rudnicka L. Effective treatment of female androgenic alopecia with dutasteride. J Drugs Dermatol. 2005;4(5):637-640.
Drug Design, Development and Therapy

\section{Publish your work in this journal}

Drug Design, Development and Therapy is an international, peerreviewed open-access journal that spans the spectrum of drug design and development through to clinical applications. Clinical outcomes, patient safety, and programs for the development and effective, safe, and sustained use of medicines are a feature of the journal, which has also

\section{Dovepress}

been accepted for indexing on PubMed Central. The manuscript management system is completely online and includes a very quick and fair peer-review system, which is all easy to use. Visit http://www. dovepress.com/testimonials.php to read real quotes from published authors. 